\begin{tabular}{|c|l|}
\hline Title & U mbilicity of spacelike submanifolds of Minkowski space \\
\hline Author(s) & Izumiya, S.; Pei, S.; Fuster, M. C. Romero \\
\hline Citation & Hokkaido University Preprint Series in Mathematics, 558, 1-14 \\
\hline Issue Date & 2002-07 \\
\hline DOI & 10.14943/83703 \\
\hline Doc URL & http://hdl.handle.net/2115/69307 \\
\hline Type & bulletin (article) \\
\hline File Information & pre558.pdf \\
\hline
\end{tabular}

Instructions for use 
Umbilicity of spacelike submanifolds of Minkowski space

S. Izumiya, D. Pei and M.C. Romero-Fuster

Series \#558. July 2002 


\section{HOKKAIDO UNIVERSITY PREPRINT SERIES IN MATHEMATICS}

\#532 T. Suwa, Characteristic classes of singular varieties, 26 pages. 2001.

\#533 J. Escher, Y. Giga and K. Ito, On a limiting motion and self-intersections for the intermediate surface diffusion flow, 20 pages. 2001.

\#534 Y.-H. R. Tsai, Y. Giga and S. Osher, A level set approach for computing discontinuous solutions of a class of Hamilton-Jacobi equations, 30 pages. 2001.

\#535 A. Yamagami, On Gouvêa's conjecture in the unobstructed case, 19 pages. 2001.

\#536 A. Inoue, What does the partial autocorrelation function look like for large lags, 27 pages. 2001.

\#537 T. Nakazi and T. Yamamoto, Norm of a linear combination of two operators of a Hilbert space, 16 pages. 2001.

\#538 Y. Giga, On the two-dimensional nonstationary vorticity equations, 12 pages. 2001.

\#539 M. Jinzenji, Gauss-Manin system and the virtual structure constants, 25 pages. 2001.

\#540 H. Ishii and T.Mikami, Motion of a graph by $R$-curvature, 28 pages. 2001.

\#541 . M. Jinzenji and T. Sasaki, $\mathcal{N}=4$ supersymmetric Yang-Mills theory on orbifold- $T^{4} / \mathbf{Z}_{2}$ : higher rank case, 17 pages. 2001.

\#542 T. Nakazi, The Nevanlinna counting functions for Rudin's orthogonal functions, 7 pages. 2001.

\#543 K. Sugano, On H-separable extensions of QF-3 rings, 7 pages. 2001.

\#544 A. Arai, Non-relativistic limit of a Dirac-Maxwell operator in relativistic quantum electrodynamics, 27 pages. 2001.

\#545 O. Sawada, On time-local solvability of the Navier-Stokes equations in Besov spaces, 30 pages. 2001.

\#546 C. M. Elliott, Y. Giga, and S. Goto, Dynamic boundary conditions for Hamilton-Jacobi equations, 27 pages. 2001.

\#547 Y. Nakano, Minimizing coherent risk measures of shortfall in discrete-time models with cone constraints, 22 pages. 2002.

\#548 K. tachizawa, A generalization of the Lieb-Thirring inequalities in low dimensions, 13 pages. 2002.

\#549 T. Nakazi, Absolute values and real parts for functions in the Smirnov class, 8 pages. 2002.

\#550 T. Nakazi and T. Watanabe, Properties of a Rubin's orthogonal function which is a linear combination of two inner functions, 9 pages. 2002.

\#551 T. Ohtsuka, A level set method for spiral crystal growth, 24 pages. 2002.

\#552 M.-H. Giga and Y. Giga, Minimal vertical singular diffusion preventing overturning for the Burgers equation, 18 pages. 2002.

\#553 Y. Giga and P. Rybka, Berg's effect, 12 pages. 2002.

\#554 Y. Tonegawa, Domain dependent monotonicity formula for a singular perturbation problem, 14 pages. 2002.

\#555 S. Izumiya and N. Takeuchi, New special curves and developable surfaces, 10 pages. 2002.

\#556 S. Izumiya, K. Saji and N. Takeuchi, Singularities of line congruences, 17 pages. 2002.

\#557 S. Izumiya, D. Pei and M. C. Romero Fuster, The lightcone Gauss map of a spacelike surface in Minkowski 4-space, 21 pages. 2002. 


\title{
Umbilicity of spacelike submanifolds of Minkowski space
}

\author{
S. Izumiya, D. Pei and M.C. Romero-Fuster *
}

\begin{abstract}
We study some properties of spacelike submanifolds in Minkowski nspace all whose points are umbilic with respect to some normal field. As a consequence of these and some results contained in [1] we obtain that being $\nu$-umbilic with respect to a parallel lightlike normal field implies conformal flatness for submanifolds of dimension $n-2 \geq 3$. In the case of surfaces we relate the umbilicity condition to that of total semiumbilicity (degeneracy of the curvature ellipse at every point). Moreover, if the considered normal field is parallel we show that it is everywhere timelike, spacelike or lightlike if and only if the surface is included in a hyperbolic 3-space, a de Sitter 3-space or a 3-dimensional light cone respectively. We also give characterizations of total semiumbilicity for surfaces contained in hyperbolic 4-space, de Sitter 4-space and 4-dimensional light cone.
\end{abstract}

\section{Introduction}

A submanifold $M$ immersed in Minkowski space $\mathbb{R}_{1}^{n}$ is said to be totally umbilic if $I I=\lambda I$, for some smooth function $\lambda$ on $M$, where $I$ and $I I$ respectively denote the first and second fundamental forms on $M$. The study of conditions implying umbilicity of spacelike hypersurfaces in Minkowski space is well spread in the mathematical literature. For instance, it is known that the only constant mean curvature compact spacelike hypersurfaces in the de Sitter space are the umbilical ones (see, S. Montiel (1988) [7], or [2] for a review of the results related to this). A weaker property to be considered in the case of submanifolds of codimension at least two is that of umbilicity with respect to a given normal field. That is, $I I_{\nu}=\lambda I_{\nu}$, where $\nu$ is some normal field on $M$ and $I I_{\nu}$ is the second fundamental form in the direction determined by $\nu$.

${ }^{*}$ Work partially supported by DGCYT grant no. BFM2000-1110 
It is a well known fact ([3]) that a $(n-2)$-submanifold of euclidean $n$-space is contained in a standard $(n-1)$-sphere if and only if it is umbilical with respect to some parallel normal field (that happens to be the restriction of the radial field). We consider here, in first place, the corresponding problem for spacelike $(n-2)$-submanifolds immersed in Minkowski $n$-space. We show that umbilicity with respect to a parallel pseudonormal field $\nu$ implies lying in hyperbolic $(n-1)$ space, de Sitter $(n-1)$-space or $(n-1)$-dimensional lightcone according $\nu$ is timelike, spacelike or lightlike.

An equivalent condition to umbilicity with respect to a given normal field on surfaces in euclidean 4-space is the total semiumbilicity ([10]). This is defined in terms of the curvature ellipse at each point introduced in [8] (see also [6]). Totally semiumbilical surfaces are those at which the curvature ellipse degenerates into a segment at every point except perhaps at isolated ones (umbilics) at which it becomes a point. Totally umbilical surfaces are a degenerate case for which the curvature ellipse degenerates everywhere into a point.

The concept of curvature ellipses for spacelike surfaces in Minkowski 4-space was introduced in [5]. We use here this setting in order to analyze the total semiumbilicity of such surfaces. We prove that analogously to the euclidean case this property is equivalent to umbilicity with respect to some normal field $\nu$ whose geometrical characterization is given. As a consequence of this, we show that a surface $M$ is contained in hyperbolic 3-space (de Sitter 3-space or 3-dimensional lightcone resp.) if and only if $M$ is totally semiumbilical and the curvature ellipses define parallel spacelike (timelike or lightlike resp.) normal fields over $M$. We also characterize the semiumbilicity condition for surfaces in $n$-dimensional Minkowski space, $n>4$, in particular for those contained in the 4-dimensional hyperbolic spaces, de Sitter spaces and lightcones.

\section{Basic concepts and notations}

We consider the $(n+1)$-dimensional Minkowski space $\left(\mathbb{R}^{n+1},\langle\rangle,\right)$, with the pseudo scalar product given by

$$
\left\langle\left(x_{0}, x_{1}, \ldots, x_{n}\right),\left(y_{0}, y_{1}, \ldots, y_{n}\right)\right\rangle=-x_{0} y_{0}+x_{1} y_{1}+\ldots+x_{n} y_{n} .
$$

We shall denote this space by $\mathbb{R}_{1}^{n+1}$.

We say that a vector $\mathrm{x}=\left(x_{0}, \ldots, x_{n}\right) \in \mathbb{R}_{1}^{n+1} \backslash\{0\}$ is spacelike, timelike or lightlike provided $\langle\mathbf{x}, \mathbf{x}\rangle>0,=0$ or $<0$ respectively. The norm or length of a vector $\mathrm{x} \in \mathbb{R}_{1}^{4}$ is defined by $\|\mathbf{x}\|=(|\langle\mathbf{x}, \mathbf{x}\rangle|)^{\frac{1}{2}}$.

Given a vector $\mathbf{v} \in \mathbb{R}_{1}^{n+1}$ and a real number $c$, we define the hyperplane with pseudonormal $\mathbf{v}$ as

$$
H P(\mathbf{v}, c)=\left\{\mathbf{x} \in \mathbb{R}_{1}^{n+1} \mid\langle\mathbf{x}, \mathbf{v}\rangle=c\right\} .
$$

This hyperplane is said to be spacelike, timelike or lightlike according to $\mathbf{v}$ is timelike, spacelike or lightlike. 
We define the Hyperbolic n-space by

$$
H_{+}^{n}(-1)=\left\{\mathbf{x} \in \mathbb{R}^{n+1} \mid\langle\mathbf{x}, \mathbf{x}\rangle=-1, x_{0} \geq 1\right\}
$$

and the de Sitter n-space by

$$
S_{1}^{n}=\left\{\mathbf{x} \in \mathbb{R}^{n+1} \mid\langle\mathbf{x}, \mathbf{x}\rangle=1\right\} .
$$

The Hyperbolic n-space and de Sitter $n$-space with center $a \in \mathbb{R}_{1}^{n+1}$ and radius $r \in \mathbb{R}_{+}$are respectively defined by

$$
\begin{gathered}
H_{+}^{n}(a ; r)=\left\{\mathbf{x} \in \mathbb{R}^{n+1} \mid\langle\mathbf{x}-\mathbf{a}, \mathbf{x}-\mathbf{a}\rangle=-r, x_{0} \geq 1\right\}, \\
S_{1}^{n}(a ; r)=\left\{\mathbf{x} \in \mathbb{R}^{n+1} \mid\langle\mathbf{x}-\mathbf{a}, \mathbf{x}-\mathbf{a}\rangle=r\right\} .
\end{gathered}
$$

We also consider the set

$$
L C_{a}=\left\{\mathrm{x} \in \mathbb{R}^{n+1} \mid\langle\mathbf{x}-\mathbf{a}, \mathbf{x}-\mathbf{a}\rangle=0\right\}
$$

known as the $n$-dimensional light cone with vertex a.

Suppose that $M$ is a surface immersed in $\mathbb{R}_{1}^{n+1}$. We say that $M$ is a spacelike surface if the tangent plane $T_{p} M$ is spacelike (i.e., consists of spacelike vectors) and thus a euclidean plane $\left(T_{p} M,\langle\rangle,\right)$ for every point $p \in M$. In this case, the normal space $N_{p} M$ is a Lorentz $(n-1)$-space $\left(\left(N_{p} M,\langle\rangle,\right)\right.$.

\section{Principal configurations associated to normal vector fields}

Given a smooth oriented surface $M$ immersed in $\mathbb{R}_{1}^{n+1}$, we denote by $\mathcal{X}(M)$ and $\mathcal{N}(M)$ the space of the smooth vector fields tangent to $M$ and the space of the smooth vector fields normal to $M$, respectively. Consider the second fundamental map,

$$
\alpha: \mathcal{X}(M) \times \mathcal{X}(M) \rightarrow \mathcal{N} M, \alpha(X, Y)=\bar{\nabla}_{\bar{X}} \bar{Y}-\nabla_{X} Y,
$$

where $\bar{\nabla}$ denotes the pseudoriemannian connection of $\mathbb{R}_{1}^{n+1}$ and $\bar{X}$ and $\bar{Y}$ are local extensions to $\mathbb{R}_{1}^{n+1}$ of tangent vector fields $X$ and $Y$ on $M$. This map is well defined, symmetric and bilinear. Given any normal field $\nu \in \mathcal{N}(M) \backslash\{0\}$, we have for each $\mathbf{x} \in M$ a function

$$
H_{\nu}: T_{\mathbf{x}} M \times T_{\mathbf{x}} M \rightarrow \mathbb{R}, H_{\nu}(v, w)=\langle\alpha(v, w), \nu(\mathbf{x})\rangle .
$$

which is also symmetric and bilinear. The second fundamental form of $M$ at $\mathbf{x}$ is the associated quadratic form,

$$
I I_{\nu}: T_{\mathbf{x}} M \rightarrow \mathbb{R}, I I_{\nu}(v)=H_{\nu}(v, v) .
$$


Then the shape operator associated to the normal field $\nu$ is defined as

$$
S_{\nu}: T M \rightarrow T M, S_{\nu}(X)=-\left(\bar{\nabla}_{\bar{X}} \bar{\nu}\right)^{\top},
$$

where $\bar{\nu}$ is a local extension to $\mathbb{R}^{n+1}$ of the normal vector field $\nu$ at $\mathbf{x}$ and ()$^{\top}$ means the tangent component. This operator is bilinear, self-adjoint and satisfies the following equation: $\left\langle S_{\nu}(X), Y\right\rangle=H_{\nu}(X, Y), \forall X, Y \in \mathcal{X}(M)$. So, we have that $I I_{\nu}(X)=\left\langle S_{\nu}(X), X\right\rangle$.

We can find for each $\mathrm{x} \in M$, an orthonormal basis of eigenvectors of $S_{\nu} \in T_{\mathrm{x}} M$, for which the restriction of the second fundamental form to the unitary vectors, $\left.I I_{\nu}\right|_{S^{1}}$, takes its maximal and minimal values. The corresponding eigenvalues $k_{1}, k_{2}$ are the $\nu$-principal curvatures, respectively. A point $\mathbf{x}$ is said to be $\nu$-umbilic if both $\nu$-principal curvatures coincide at $\mathbf{x}$. Let $\mathcal{U}_{\nu}$ be the set of $\nu$-umbilics in $M$. For any $\mathbf{x} \in M \backslash \mathcal{U}_{\nu}$ there are two $\nu$-principal directions defined by the eigenvectors of $S_{\nu}$, these fields of directions are smooth and integrable, then they define two families of orthogonal curves, its integrals, which are called the $\nu$-principal lines of curvature. The two orthogonal foliations with the $\nu$-umbilics as its singularities form the $\nu$-principal configuration of $M$. We say that the surface $M$ is $\nu$-umbilical if each point of $M$ is $\nu$-umbilic.

The differential equation of $\nu$-lines of curvature is

$$
S_{\nu}(X(p))=\lambda(p) X(p)
$$

Suppose that $M$ is locally given by an immersion $\mathbf{X}: U \rightarrow \mathbb{R}_{1}^{n+1}$, with local coordinates $(u, v)$. Let $E, F, G$ be the coefficients of the first fundamental form in this coordinate chart. The coefficients of the second fundamental form are given by

$$
\begin{aligned}
& e_{\nu}=I I_{\nu}\left(\partial_{u}\right)=-\left\langle\alpha\left(\partial_{u}, \partial_{u}\right), \nu\right\rangle=\left\langle\mathbf{X}_{u u}, \nu\right\rangle, \\
& f_{\nu}=-\left\langle\alpha\left(\partial_{u}, \partial_{v}\right), \nu\right\rangle=-\left\langle\alpha\left(\partial_{v}, \partial_{u}\right), \nu\right\rangle=\left\langle\mathbf{X}_{u v}, \nu\right\rangle, \\
& g_{\nu}=I I_{\nu}\left(\partial_{v}\right)=-\left\langle\alpha\left(\partial_{v}, \partial_{v}\right), \nu\right\rangle=\left\langle\mathbf{X}_{v v}, \nu\right\rangle,
\end{aligned}
$$

where $\partial_{u}=\frac{\partial}{\partial u}, \partial_{v}=\frac{\partial}{\partial v}, \mathbf{X}_{u u}=\frac{\partial^{2} \mathbf{X}}{\partial u^{2}}, \mathbf{X}_{u v}=\frac{\partial^{2} \mathbf{X}}{\partial u \partial v}$ and $\mathbf{X}_{u u}=\frac{\partial^{2} \mathbf{X}}{\partial v^{2}}$.

Equation (1) has the following expression in this coordinate chart, [9].

$$
\left(f_{\nu} E-e_{\nu} F\right) d u^{2}+\left(g_{\nu} E-e_{\nu} G\right) d u d v+\left(g_{\nu} F-f_{\nu} G\right) d v^{2}=0 .
$$

If we assume that this coordinate chart is isothermic, so $E=G>0, F=0$, this equation has the form

$$
f_{\nu} d u^{2}+\left(g_{\nu}-e_{\nu}\right) d u d v-f_{\nu} d v^{2}=0,
$$

Remark 3.1 If we take coordinates such that the immersion $\mathbf{X}$ above is given in the Monge form, it can be easily seen that the matrix of the quadratic form $I I_{\nu}$ (and thus that of the operator $S_{\nu}$ ) at a point $p$ coincides with that of the hessian of the height function $\mathbf{X}_{\nu(p)}$ at $p$. 


\section{$4 \quad \nu$-umbilicity.}

It is known that surfaces immersed in a 3-sphere of euclidean 4-space are totally umbilic with respect to the radial field $([3],[10])$. We see next that the analogous statement for surfaces immersed in Minkowski $n$-space is also true.

Lemma 4.1 Let $M$ be a surface immersed in either $H^{n-1}(a ;-r), S^{n-1}(a ; r)$ or $L C_{a}$, for some $a \in \mathbb{R}_{1}^{n}$ and some $r \in \mathbb{R}_{+}$. Then $M$ is $\nu$-umbilic, where $\nu$ is the position field at each point of $M$.

Proof: Suppose that $M$ is locally given by $\mathbf{X}: U \rightarrow \mathbb{R}_{1}^{n}$ as above, so $\nu=\mathbf{X}$. In each one of the three considered cases we have that $\langle\mathbf{X}, \mathbf{X}\rangle$ is constant. Hence, $\left\langle\mathbf{X}_{u}, \mathbf{X}\right\rangle=\left\langle\mathbf{X}_{v}, \mathbf{X}\right\rangle=0$.

By considering isothermic coordinates we have,

$$
\begin{aligned}
& e_{\nu}=\left\langle\mathbf{X}_{u u}, \nu\right\rangle=-\left\langle\mathbf{X}_{u}, \mathbf{X}_{u}\right\rangle=-E, \\
& f_{\nu}=\left\langle\mathbf{X}_{u v}, \nu\right\rangle=-\left\langle\mathbf{X}_{u}, \mathbf{X}_{v}\right\rangle=0, \\
& g_{\nu}=\left\langle\mathbf{X}_{v v}, \nu\right\rangle=-\left\langle\mathbf{X}_{v}, \mathbf{X}_{v}\right\rangle=-G=-E .
\end{aligned}
$$

Therefore, $e_{\nu}=g_{\nu}$ and $f_{\nu}=0$ at each point and it follows from equation (2) that the surface is $\nu$-umbilic.

Suppose that $M$ is a spacelike $(n-2)$-manifold locally given by an immersion $\mathbf{X}: U \rightarrow \mathbb{R}_{1}^{n}$. Then we can choose a pseudo-orthonormal frame $\left\{e_{1}, e_{2}\right\}$ for the normal bundle and an orthonormal frame $\left\{e_{3}, \ldots, e_{n}\right\}$ for the tangent bundle of $M$, with $e_{1}$ timelike and $e_{i}, i=2, \ldots, n$ spacelike vectors. We have then the dual 1-forms $\omega_{i}=\delta\left(e_{i}\right)\left\langle d \mathbf{X}, e_{i}\right\rangle$ and the connection 1-forms $\omega_{i j}=\delta\left(e_{i}\right)\left\langle d e_{i}, e_{j}\right\rangle$, where

$$
\delta\left(e_{i}\right)=\operatorname{Sign}\left(e_{i}\right)= \begin{cases}1 & i=2, \ldots, n \\ -1 & i=1\end{cases}
$$

Lemma 4.2 Suppose that $M$ is an (n-2)-submanifold immersed in Minkowski $n$-space that is $\nu$-umbilical for some constant length normal field $\nu$ on $M$ with curvature function $\lambda$. Then we have,

a) If $\nu$ is parallel then $\lambda$ is constant.

b) Supposing that $\nu$ is parallel, then $\lambda=0$ if and only if $\nu$ is a constant field.

Proof: We may have that $\nu$ is either everywhere timelike, spacelike lightlike. Suppose first that $\nu$ is a timelike parallel normal field. Moreover, since $\nu$ has constant length, we may assume that $\langle\nu, \nu\rangle=-1$. In this case we may choose $e_{1}=\nu$. We then observe that the parallelism condition on $\nu$ is equivalent to $\omega_{12}=0$. We prove now that $\omega_{12}=0 \Rightarrow \lambda=$ constant. In fact, we have

$$
\begin{aligned}
\omega_{1 j}(X)= & \left\langle\bar{\nabla}_{X} \bar{e}_{1}, e_{j}\right\rangle=\left\langle S_{e_{1}}(X)+\bar{\nabla}_{X}^{\frac{1}{e_{e}}} \bar{e}_{1}, e_{j}\right\rangle=\left\langle S_{e_{1}}(X), e_{j}\right\rangle \\
& =\left\langle\lambda X, e_{j}\right\rangle=\lambda \omega_{j}(X), \quad j=3, \ldots, n, \forall X \in \mathcal{X}(M) .
\end{aligned}
$$


So $\omega_{1 j}=\lambda \omega_{j}, j=3, \ldots, n$ and by taking exterior derivatives

$$
d \lambda \wedge w_{j}+\lambda d w_{j}=d w_{1 j}, \quad j=3, \ldots, n .
$$

Take $j=3$ and consider the structure equations ([5], [11])

$$
d w_{13}=w_{12} \wedge w_{23}+w_{14} \wedge w_{43}+\ldots+w_{1 n} \wedge w_{n 3} .
$$

So we have

$$
d w_{13}=w_{12} \wedge w_{23}+\lambda\left(w_{4} \wedge w_{43}+\ldots+w_{1 n} \wedge w_{n 3} .\right.
$$

Moreover, $d \omega_{3}=\delta\left(e_{3}\right) \delta\left(e_{4}\right) \omega_{34} \wedge \omega_{4}+\ldots+\delta\left(e_{3}\right) \delta\left(e_{n}\right) \omega_{3 n} \wedge \omega_{n}=\omega_{4} \wedge \omega_{43}+\ldots+$ $\omega_{n} \wedge \omega_{n 3}$. Therefore,

$$
d \lambda \wedge \omega_{3}+\lambda d \omega_{3}=d \omega_{13}=\omega_{12} \wedge \omega_{23}+\lambda \omega_{4} \wedge \omega_{43} .
$$

And hence,

$d \lambda \wedge \omega_{3}+\lambda\left(\omega_{4} \wedge \omega_{43}+\ldots+\omega_{n} \wedge \omega_{n 3}\right)=\omega_{12} \wedge \omega_{23}+\lambda\left(\omega_{4} \wedge \omega_{43}+\ldots+\omega_{n} \wedge \omega_{n 3}\right)$.

Consequently,

$$
d \lambda \wedge \omega_{3}=\omega_{12} \wedge \omega_{23}
$$

Analogous arguments for $j=4, \ldots, n$ lead to

$$
d \lambda \wedge \omega_{j}=\omega_{12} \wedge \omega_{2 j}, j=3, \ldots, n .
$$

And it clearly follows that $\omega_{12}=0 \Rightarrow d \lambda=0 \Rightarrow \lambda=$ constant.

If $\nu$ is a spacelike normal field, we take $e_{2}=\nu$. We have again that the parallelism of $\nu$ is equivalent to $\omega_{12}=0$ and an analogous argument (interchanging the subindecies 1 and 2) leads to the required result.

Suppose now that $\nu$ is a parallel lightlike vectorfield. We consider a pseudoorthonormal frame $\left\{e_{1}, e_{2}\right\}$ on $N M$ and an orthonormal frame $\left\{e_{3}, \ldots, e_{n}\right\}$ on $T M$ as above. We have that $\langle\nu, \nu\rangle=0$ and thus we can take $\nu=e_{1} \pm e_{2}$. Moreover, since $\nu$ is parallel, $\nabla_{X}^{\frac{1}{X}}\left(e_{1} \pm e_{2}\right)=0, \forall X \in \mathbf{X}(M)$. That is, $\omega_{12}=$ $\mp \omega_{11}=0$. On the other hand,

$$
\begin{aligned}
\left(\omega_{j 1} \pm \omega_{j 2}\right)(X)= & \left\langle\bar{\nabla}_{X} \bar{e}_{1} \pm \bar{\nabla}_{X} \bar{e}_{2}, e_{j}\right\rangle=\left\langle\vec{\nabla}_{X} \bar{\nu}, e_{j}\right\rangle \\
& =\left\langle S_{\nu}(X), e_{j}\right\rangle=\lambda \omega_{j}(X), \quad j=3, \ldots, n, \forall X \in \mathcal{X}(M) .
\end{aligned}
$$

Therefore, $\omega_{j 1} \pm \omega_{j 2}=\lambda \omega_{j}, j=3, \ldots, n$.

By taking derivatives we get

$$
d \lambda \wedge w_{j}+\lambda d w_{j}=d \omega_{j 1} \pm d \omega_{j 2}, \quad j=3, \ldots, n .
$$


Now, from the structure equations

$$
\begin{aligned}
& d w_{31}=w_{32} \wedge w_{21}+w_{34} \wedge w_{41}+\ldots+w_{3 n} \wedge w_{n 1} . \\
& d w_{32}=w_{31} \wedge w_{12}+w_{34} \wedge w_{42}+\ldots+w_{3 n} \wedge w_{n 2} .
\end{aligned}
$$

and taking $j=3$ in the above expression, we obtain

$$
\begin{aligned}
d \lambda \wedge w_{3}+\lambda d w_{3}= & w_{32} \wedge w_{21}+w_{34} \wedge w_{41}+\ldots+w_{3 n} \wedge w_{n 1} \\
& \pm\left(w_{31} \wedge w_{12}+w_{34} \wedge w_{42}+\ldots+w_{3 n} \wedge w_{n 2}\right) .
\end{aligned}
$$

And since $d \omega_{3}=\omega_{34} \wedge \omega_{4}+\ldots+\omega 3 n \wedge \omega_{n}$, it follows,

$d \lambda \wedge \omega_{3}+\lambda\left(\omega_{34} \wedge \omega_{4}+\ldots+\omega_{3 n} \wedge \omega_{n}\right)=\omega_{32} \wedge \omega_{21} \pm\left(w_{31} \wedge w_{12}+\left(\omega_{34} \wedge \omega_{41}+\right.\right.$ $\left.\ldots+\omega_{3 n} \wedge \omega_{n 1}\right) \pm\left(\omega_{34} \wedge \omega_{42}+\ldots+\omega_{3 n} \wedge \omega_{n 2}\right)=\omega_{12} \wedge \omega_{32} \mp \omega_{31} \wedge \omega_{12}+\omega_{34} \wedge$ $\left(\omega_{41} \pm \omega_{42}\right)+\ldots+\omega_{3 n} \wedge\left(\omega_{n 1} \pm \omega_{n 2}\right)$.

Therefore,

$$
d \lambda \wedge \omega_{3}=\omega_{32} \wedge \omega_{21} \pm \omega_{31} \wedge \omega_{12}=\omega_{12} \wedge\left(\omega_{32} \mp \omega_{31}\right) .
$$

Analogous arguments for $j=4, \ldots, n$ lead to

$$
d \lambda \wedge \omega_{4}=\omega_{12} \wedge\left(\omega_{j 2} \mp \omega_{j 1}\right) .
$$

And we have that $\omega_{12}=0 \Rightarrow d \lambda=0 \Rightarrow \lambda=$ constant, in this case too. So statement a) is shown.

b) Suppose now that $\nu$ is constant. Again, if $\nu$ is timelike, we can put $e_{1}=\nu$ and we have that $\bar{\nabla}_{X} \bar{e}_{1}=0, \forall X \in \mathcal{X}(M)$. In particular, $\omega_{1} j(X)=\left\langle\bar{\nabla}_{X} \bar{e}_{1}, e_{j}\right\rangle=$ $0, \forall X, j=3, \ldots, n$. But as we have seen before, $\omega_{1 j}=\lambda \omega_{j}, j=3, \ldots, n$. So it follows that $\lambda=0$. The argument for a spacelike $\nu$ runs analogously. On the other hand, if $\nu$ is a constant lightlike normal field, we can put $\nu=e_{1} \pm e_{2}$. We then have that $\bar{\nabla}_{e_{j}}\left(\bar{e}_{1} \pm \bar{e}_{2}\right)=0, j=3, \ldots, n$. Therefore, $\omega_{1 j} \pm \omega_{2 j}=0$. But in this case, as we have seen above, $\omega_{1 j} \pm \omega_{2 j}=\lambda \omega_{j}, j=3, \ldots, n$ and hence $\lambda=0$.

Finally, if $\nu$ is a parallel timelike field we have that $\omega_{12}=0$ and then $\lambda=0$ implies that $\omega_{1 j}=\lambda \omega_{j}=0, j=3, \ldots, n$. Therefore, $\left\langle\bar{\nabla}_{X} \bar{e}_{1}, e_{j}\right\rangle=0, \forall X, j=$ $2,3, \ldots, n$. And thus, $\bar{\nabla}_{X} \bar{e}_{1}=0, \forall X \in \mathcal{X}(M)$, which implies that $\nu=e_{1}$ is constant. The spacelike and lightlike cases are proven in a similar way.

Theorem 4.3 Let $M$ be a $(n-2)$-submanifold immersed in $\mathbb{R}_{1}^{n}$.

i) If $M$ is $\nu$-umbilic for some parallel everywhere timelike field $\nu$ then either $M$ is contained in some hyperbolic 3-space, provided $\nu$ has nonzero curvature, or $M$ lies in a spacelike hyperplane in case that $\nu$ has vanishing curvature.

ii) If $M$ is $\nu$-umbilic for some parallel everywhere spacelike field $\nu$ then either $M$ is contained in some de Sitter 3-space, provided $\nu$ has nonzero curvature, or $M$ lies in a timelike hyperplane in case that $\nu$ has vanishing curvature.

iii) If $M$ is $\nu$-umbilic for some parallel everywhere lightlike field $\nu$ then either $M$ is contained in some light cone, provided $\nu$ has nonzero curvature, or $M$ lies in a lightlike hyperplane in case that $\nu$ has vanishing curvature. 
Proof: Suppose that $M$ is $\nu$-umbilical and hence, for any vector $X$ tangent to $M$, we have

$$
\bar{\nabla}_{X} \nu=\nabla_{X} \nu+\bar{\nabla}_{X}^{\perp} \nu=\lambda X+\bar{\nabla}_{X}^{\perp} \nu
$$

But $\bar{\nabla}^{\perp} \nu=0$, for $\nu$ is parallel, and thus

$$
\bar{\nabla}_{X} \nu=\lambda X
$$

On the other hand, we have that the covariant derivation of the radial vector field $\mathbf{X}$ is the identity, i.e., $\nabla_{X} \mathbf{X}=\mathbf{X}$, for any vector $\mathbf{X}$ tangent to $\mathbb{R}_{1}^{n}$. Therefore the following equation holds:

$$
\vec{\nabla}_{X}(\lambda \mathbf{X}-\nu)=0
$$

for any $X$ tangent to $M$. It follows that $\nu-\lambda \mathrm{X}$ is a constant vector $X_{0}$, so

$$
\lambda \mathbf{X}(p)-\nu(p)=X_{0}, \forall p \in M .
$$

Now, in case that $\lambda \neq 0$ we can put

$$
\mathbf{X}(p)-\frac{X_{0}}{\lambda}=\frac{\nu(p)}{\lambda}, \forall p \in M
$$

which, being $\lambda$ constant, means that $M$ belongs to either a hyperbolic $(n-1)$ space in case that $\nu$ is spacelike, to a de Sitter $(n-1)$-space in case that it is timelike, or to a lightcone in case that it is lightlike.

If $\lambda=0$ we have from lemma 4.2 that $\nu$ must be a constant normal field $X_{0}$ over $M$. But this implies that $M$ is contained in a hyperplane orthogonal to $X_{0}$.

It has been shown in [1] that for a Riemannian $(n-2)$-manifold with $n \geq 5$, the condition of being conformally flat is equivalent to admit some isometric embedding in the lightcone of a Minkowski $n$-space. Consequently, we have

Corollary 4.4 If $M$ is a $(n-2)$-submanifold of $\mathbb{R}_{1}^{n}, n \geq 3$ which is umbitic with respect to some parallel lightlike normal field $\nu$ then $\bar{M}$ is conformally flat.

Remark 4.5 The computations made in lemma 4.2 can be easily adapted to the case of higher codimensional spacelike submanifolds of $\mathbb{I R}_{1}^{n}$. So the results established in theorem 4.3 also hold for these submanifolds.

As a consequence of lemma 4.1 and theorem 4.3 for surfaces in $\mathbb{R}_{1}^{4}$ we have the following corollary:

Corollary 4.6 Let $M$ be a surface immersed in $\mathbb{R}_{1}^{4}$.

i) $M$ is $\nu$-umbilic for some parallel everywhere timelike field $\nu$ if and only if either $M$ is contained in some hyperbolic 3-space, provided $\nu$ has nonzero 
curvature, or $M$ lies in a spacelike hyperplane in case that $\nu$ has vanishing curvature.

ii) $M$ is $\nu$-umbilic for some parallel everywhere spacelike field $\nu$ if and only if either $M$ is contained in some de Sitter 3-space, provided $\nu$ has nonzero curvature, or $M$ lies in a timelike hyperplane in case that $\nu$ has vanishing curvature.

iii) $M$ is $\nu$-umbilic for some parallel everywhere lightlike field $\nu$ if and only if either $M$ is contained in some light cone, provided $\nu$ has nonzero curvature, or $M$ lies in a lightlike hyperplane in case that $\nu$ has vanishing curvature.

\section{$5 \quad$ Semiumbilicity}

Given a surface $M$ immersed in $\mathbb{R}_{1}^{n+1}$ consider local isothermic coordinates $\{x, y\}$ on $M$ and a pseudo-orthonormal frame, $\left\{e_{1}, e_{2}, \cdots, e_{n+1}\right\}$ in a neighbourhood of $\mathbf{x}=f(0,0) \in M$, such that $\left\{e_{2}, \ldots, e_{n+1}\right\}$ is a normal frame and $\left\{e_{n}, e_{n+1}\right\}$ a tangent frame, with $\left\langle e_{1}, e_{1}\right\rangle=-1$ and $\left\langle e_{i}, e_{i}\right\rangle=1, i=2, \ldots, n+1$. The matrix of the bilinear form $H_{e_{i}}$ is given by

$$
H_{e_{i}}(\mathbf{x})=\left[\begin{array}{cc}
a_{i} & b_{i} \\
b_{i} & c_{i}
\end{array}\right]
$$

where if $d s^{2}=E\left(d x^{2}+d y^{2}\right)$ is the firat fundamental form, we have

$$
a_{i}=\frac{1}{E} \frac{\partial^{2} f}{\partial x^{2}}(0,0) \cdot e_{i}, \quad b_{i}=\frac{1}{E} \frac{\partial^{2} f}{\partial x \partial y}(0,0) \cdot e_{i}, \quad c_{i}=\frac{1}{E} \frac{\partial^{2} f}{\partial y^{2}}(0,0) \cdot e_{i},
$$

for $i=1, \cdots, n-1$.

Given $\mathrm{x} \in M$, consider the unit circle in $T_{\mathrm{x}} M$ parameterized by the angle $\theta \in[0,2 \pi]$. Denote by $\gamma_{\theta}$ the spacelike curve obtained by intersecting $M$ with the timelike hyperplane defined by the direct sum of the normal subspace $N_{\mathbf{x}} M$ and the straight line in the tangent direction represented by $\theta$. Such curve is called the normal section of $M$ in the tangent direction $\theta$. The curvature vector $\eta(\theta)$ of $\gamma_{\theta}$ in $\mathbf{x}$ lies in the timelike hyperplane $N_{\mathbf{x}} M$. Varying $\theta$ from 0 to $2 \pi$, the vector $\eta(\theta)$ describes an ellipse in $N_{\mathbf{x}} M$, called the curvature ellipse of $M$ at $\mathbf{x}$. It can be seen that the curvature ellipse is the image of the affine map (we refer to [5] for the case $n=3$, the case $n \geq 4$ is a straightforward generalization)

$$
\eta: S^{1} \subset T_{\mathbf{x}} M \longrightarrow N_{\mathbf{x}} M
$$

given by

$$
\theta \longmapsto \eta(\theta)=\sum_{i=1}^{n-1}\left[\begin{array}{cc}
\cos \theta & \sin \theta
\end{array}\right] \cdot\left[\begin{array}{cc}
a_{i} & b_{i} \\
b_{i} & c_{i}
\end{array}\right] \cdot\left[\begin{array}{c}
\cos \theta \\
\sin \theta
\end{array}\right] \cdot e_{i},
$$

that is, 


$$
\eta(\theta)=H_{\mathbf{x}}+B_{\mathbf{x}} \cos 2 \theta+C_{\mathbf{x}} \sin 2 \theta,
$$

with

$$
\begin{gathered}
H_{\mathbf{x}}=\frac{1}{2}\left(a_{1}+c_{1}\right) e_{1}-\frac{1}{2} \sum_{i=2}^{n-1}\left(a_{i}+c_{i}\right) \cdot e_{i}, \\
B_{\mathbf{x}}=\frac{1}{2}\left(a_{1}-c_{1}\right) e_{1}-\sum_{i=2}^{n-1}\left(a_{i}-c_{i}\right) \cdot e_{i}, \\
C_{\mathbf{x}}=b_{1} e_{1}-\sum_{i=2}^{n-1} b_{i} \cdot e_{i} .
\end{gathered}
$$

This ellipse, contained in the Lorentz $(n-1)$-space $N_{\mathbf{x}} M$, may degenerate into a segment for certain points of $M$ that we call semiumbilic points. A semiumbilic point $\mathbf{x}$ is said to be spacelike, timelike or lightlike provided the curvature segment defines respectively a spacelike, timelike or lightlike direction in $N_{\mathrm{x}} M$. There may be also points at which the curvature ellipse becomes a point, these are degenerate semiumbilics called umbilics. A surface all whose points are semiumbilic (except by isolated umbilics) is said to be totally semiumbilical.

Proposition 5.1 Let $M$ be a spacelike surface immersed in $\mathbb{R}_{1}^{n+1}$ and let $\nu \in$ $\mathcal{N}(M) \backslash\{0\}$. A point $\mathbf{x} \in M$ is $\nu$-umbilic if and only if $\nu(\mathbf{x})$ is pseudo-orthogonal to the vectors $B_{\mathbf{x}}$ and $C_{\mathbf{x}}$ defined above.

Proof: With the same notation as the above we can write $\nu=\nu_{1} e_{1}+\ldots+$ $\nu_{n-1} e_{n-1}$. Then $e_{\nu}=-\sum_{i=1}^{n-1} \nu_{i} a_{i}, f_{\nu}=-\sum_{i=1}^{n-1} \nu_{i} b_{i}$ and $g_{\nu}=-\sum_{i=1}^{n-1} \nu_{i} c_{i}$. So it follows from the expression (2) that $\mathrm{x}$ is a $\nu$-umbilic point if and only if the following two functions

$$
\begin{gathered}
f_{\nu}=-\sum_{i=1}^{n-1} \nu_{i} b_{i} \\
e_{\nu}-g_{\nu}=-\sum_{i=1}^{n-1} \nu_{i}\left(a_{i}-c_{i}\right)
\end{gathered}
$$

vanish at $\mathbf{x}$.

On the other hand, we have that

$$
\begin{gathered}
\left\langle\nu, C_{\mathbf{x}}\right\rangle=-\sum_{i=1}^{n-1} \nu_{i} b_{i} \\
\left\langle\nu, B_{\mathbf{x}}\right\rangle=-\sum_{i=1}^{n-1} \nu_{i}\left(a_{i}-c_{i}\right)
\end{gathered}
$$

which leads to the required result. 
Remark 5.2 It follows from the above proposition that the umbilic points of $M$ are critical points of all the principal configurations defined by normal fields on $M$.

Theorem 5.3 A spacelike surface $M \subset \mathbb{R}_{1}^{4}$ is totally semiumbilical if and only if $M$ is $\nu$-umbilical for some $\nu \in \mathcal{N}(M) \backslash\{0\}$ locally defined at each non umbilical point.

Proof: Suppose that $M$ is totally semiumbilical, so the ellipse is a segment at every point in a neighbourhood of any non umbilic point $\mathrm{x} \in M$, we can define a normal field $\nu$ locally given by the pseudo-orthogonal direction to that of the curvature segment in this neighbourhood. Then it follows from proposition 5.1 that every point of $M$ where $\nu$ is defined is $\nu$-umbilic.

Conversely, we have that $N_{\mathrm{x}} M$ is a Lorentz plane and the condition that a nonzero normal vector $\nu(\mathbf{x})$ is pseudo-orthogonal to the vectors $B_{\mathbf{x}}$ and $C_{\mathbf{x}}$ implies that these two last vectors must be parallel. Since they generate the curvature ellipse at $\mathbf{x} \in M$ we have that this ellipse degenerates.

Remark 5.4 We observe that in the proposition 5.3 the normal vector $\nu(\mathbf{x})$ is timelike, spacelike or lightlike if and only if the point $\mathrm{x}$ is a spacelike, timelike or lightlike semiumbilic respectively. Moreover, in the last case the curvature ellipse has direction $\nu(\mathbf{x})$.

Therefore, from Theorem 4.1 we get the following

Corollary 5.5 Given a spacelike surface $M \subset \mathbb{R}_{1}^{4}$ we have that

i) $M \subset H^{3}(a ;-r) \Leftrightarrow$ every point of $M$ is either spacelike semiumbilical or umbilical and the curvature ellipses define a parallel normal field on $M$.

ii) $M \subset S^{3}(a ; r) \Leftrightarrow$ every point of $M$ is either timelike semiumbilical or umbilical and the curvature ellipses define a parallel normal field on $M$.

iii) $M \subset L C^{3}(a) \Leftrightarrow$ every point of $M$ is either lightlike semiumbilical or umbilical and the curvature ellipses define a parallel normal field on $M$.

The following generalization for surfaces immersed in higher dimensional Minkowski space can be analogously proven.

Theorem 5.6 A spacelike surface $M \subset \mathbb{R}_{1}^{n}, n \geq 4$ is totally semiumbilical if and only if there exist linearly independent normal fields $\nu_{1}, \nu_{3}, \ldots, \nu_{n-3}$, locally defined at every non umbilical point of $M$, such that $M$ is $\nu_{i}$-umbilical.

Corollary 5.7 A spacelike surface $M \subset \mathbb{R}_{1}^{n}, n \geq 4$ is totally semiumbilical if and only if it has a unique nontrivial principal configuration.

Proof: In virtue of theorem 5.6, if $M$ is totally semiumbilic, each non umbilic normal field $\eta$ can be written as $\eta=\lambda_{1} \nu_{1}+\ldots+\lambda_{n-3} \nu_{n-3}+\lambda_{n-2} \xi$, where $\xi$ is 
a field orthogonal to the umbilic fields $\nu_{i}, i=1, \ldots, n-3$ and $\lambda_{i}, i=1, \ldots, n-$ 2 are smooth functions and $\lambda_{n-2}$ is never vanishing. Clearly, the principal configurations associated to $\eta$ and $\xi$ coincide and the result follows.

To see the converse we observe that given two linearly independent normal fields with the same associated configurations it is always possible to find a linear combination of both with a trivial principal configuration.

We consider now the particular class of spacelike surfaces of $\mathbb{R}_{1}^{5}$ given by those contained in either the hyperbolic 4-space $H_{-}^{4}(-1)$, the de Sitter space $S_{+}^{4}(1)$ or the lightcone $L C^{4}(0)$. Their total semiumbilicity can be characterized as follows

Proposition 5.8 Given a spacelike surface $M \subset H_{+}^{4}(-1)\left(S_{+}^{4}(1)\right.$ or $L C^{4}(0)$ resp.).

a) the curvature ellipse of $M$ at a point $\mathbf{x} \in M$ is contained in $T_{\mathbf{x}} H_{+}^{4}(-1)$ $\left(T_{\mathrm{x}} S_{+}^{4}(1)\right.$ or $T_{\mathrm{x}} L C^{4}(0)$ resp. $)$.

b) $M$ is totally semiumbilical if and only if $M$ is $\nu$-umbilic, for some field $\nu \in \mathcal{N} M \cap \mathcal{X} H_{+}^{4}(-1)\left(\nu \in \mathcal{N} M \cap \mathcal{X} S_{+}^{4}(1)\right.$ or $\nu \in \mathcal{N} M \cap \mathcal{X} L C^{4}(0)$ resp. $)$.

Proof: a) It follows immediately from lemma 4.1 that the position field is totally umbilic on $M$, but then proposition 5.1 implies that it must be pseudoorthogonal to the vectors $B_{\mathrm{x}}$ and $C_{\mathrm{x}}$ that determine the plane of the curvature ellipse at $\mathbf{x}$. Since the position vector at $\mathbf{x}$ is also pseudo-orthogonal to $T_{\mathrm{x}} H^{4}(-1)\left(\right.$ resp. $T_{\mathrm{x}} S_{+}^{4}(1)$ or $\left.T_{\mathrm{x}} L C^{4}(0)\right), \forall \mathbf{x} \in M$, we have that this ellipse lies in $T_{\mathbf{x}} H^{4}(-1)$ (resp. $T_{\mathbf{x}} S_{+}^{4}(1)$ or $\left.T_{\mathbf{x}} L C^{4}(0)\right) \forall \mathbf{x} \in M$. The assertion b) is an immediate consequence of assertion a) and proposition 5.1.

Corollary 5.9 A spacelike surface $M \subset H_{+}^{4}(-1)$ is totally semiumbilical if and only if $M$ is umbilical with respect to some lightlike normal field.

Proof: We have that $M$ is umbilical with respect to the position field $\mathbf{X}$, which is timelike in this case. Moreover, given $\mathbf{x} \in M$, the plane $N_{\mathbf{x}} M \cap T_{\mathbf{x}} H^{4}(-1)$ is a euclidean plane. Then, given any nevervanishing vectorfield $\nu \in \mathcal{N} M \cap$ $\mathcal{X} H_{+}^{4}(-1)$, we can always find a lightlike linear combination $\eta$ of $\mathbf{X}$ and $\nu$. Since $M$ is $\mathbf{X}$-umbilical, we have that $M$ is $\nu$-umbilical if and only if $M$ is $\eta$-umbilical and then the result follows from part $b$ ) of proposition 5.8.

The hypersurfaces of $H_{+}^{n}(-1)$ determined by intersecting it with a spacelike, a timelike or a lightlike hyperplane are respectively called hyperspheres, equidistant hyperplanes and hyperhorospheres. The hyperhorospherical geometry of hypersurfaces in the Hyperbolic $n$-space has been studied in [4] where the invariant known the hyperbolic Gauss-Kronecker curvature was introduced. It was there shown that the vanishing of this invariant on a hypersurface implies that it is contained in a hyperhorosphere. We finally give a characterization of the fact of being contained in a hyperhorosphere for surfaces in $H_{+}^{n}(-1)$ in terms of umbilicity. 
Corollary 5.10 A spacelike surface $M \subset H_{+}^{n}(-1)$ lies in a hyperhorosphere if and only if it is umbilical with respect to some lightlike normal field $\nu$ with constant zero curvature.

Proof: Observe that in this case $\nu$ must be constant (remark 4.5) so $M$ is $\nu$-umbilical if and only if it is contained in a lightlike hyperplane and thus in a hyperhorosphere.

\section{References}

[1] A.C. Asperti and M. Dajczer, Conformally flat Riemannian manifolds as hypersurfaces of the lightcone, Canad. math. Bull. 32 (1989), 281-285.

[2] A. Brasil and G. Colares, On constant mean curvature spacelike hypersurfaces in Lorentz manifolds. Matemática Contemporânea 17 (1999), 99-136.

[3] B. Y. Chen and K. Yano, Integral formulas for submanifolds and their applications. J. Differential Geometry 5 (1971), 467-477. (?)

[4] S. Izumiya, D. Pei and T. Sano, Singularities of hyperbolic Gauss maps. To appear in Proceedings of the London Mathematical Society.

[5] S. Izumiya, D. Pei and M.C. Romero Fuster, The lightcone Gauss map of a spacelike surface in Minkowski 4-space. Preprint (2001).

[6] J. Little, On singularities of submanifolds of higher dimensional Euclidean space. Annali Mat. Pura et Appl., (ser. 4A) 83 (1969), 261-336.

[7] S. Montiel, An integral inequality for compact spacelike hypersurfaces in the de Sitter space and applications to the case of constant mean curvature. Indiana Univ. Math. J. 37 (1988), 909-917.

[8] C. L. E. Moore and E. B. Wilson, Differential Geometry of twodimensional surfaces in hyperspaces. Proc. Amer. Acad. Arts and Sciences 52 (1916), 267-368.

[9] A. Ramirez-Galarza and F. Sánchez-Bringas, Lines of Curvature near Umbilical Points on Surfaces Immersed in $\mathbb{R}^{4}$. Annals of Global Analysis and Geometry 13 (1995), 129-140. 
[10] M.C. Romero-Fuster and F. Sánchez-Bringas, Umbilicity of surfaces with orthogonal asymptotic lines in $\mathbb{I R}^{4}$. To appear in Differential Geometry and Applications.

[11] M. Spivak, A Comprehensive Introduction to Differential Geometry, Vols. 3 and 4. Publish or Perish, (1979).

Shyuichi Izumiya, Department of Mathematics, Hokkaido University, Sapporo 060-0810, Japan

e-mail:

Donghe Pei, Department of Mathematics, North East Normal University, Changchun 130024, P.R. China

e-mail:

María del Carmen Romero Fuster, Departament de Geometria i Topologia, Universitat de València, 46100 Burjassot (València), Espanya e-mail: carmen.romero@post.uv.es 